Review

\title{
Fecal Microbiota Transplantation: Expanding Horizons for Clostridium difficile Infections and Beyond
}

\author{
Thomas J. Borody ${ }^{1, *}$, Debra Peattie ${ }^{2}$ and Scott W. Mitchell ${ }^{1}$ \\ 1 Centre for Digestive Diseases, 1/229 Great North Rd, Five Dock, NSW 2046 Australia; \\ E-Mail: scott.mitchell@cdd.com.au \\ 2 Pleiades Advisors, 13 Oak Meadow Road, Lincoln, MA 01773, USA; \\ E-Mail: debra.peattie@pleiadesadvisors.com
}

* Author to whom correspondence should be addressed; E-Mail: thomas.borody@cdd.com.au; Tel.: +61-2-9713-4011; Fax: +61-2-9712-1675.

Academic Editor: Yung-Fu Chang

Received: 6 May 2015 / Accepted: 26 June 2015 / Published: 6 July 2015

\begin{abstract}
Fecal Microbiota Transplantation (FMT) methodology has been progressively refined over the past several years. The procedure has an extensive track record of success curing Clostridium difficile infection (CDI) with remarkably few adverse effects. It achieves similar levels of success whether the CDI occurs in the young or elderly, previously normal or profoundly ill patients, or those with CDI in Inflammatory Bowel Disease (IBD). While using FMT to treat CDI, however, we learned that using the procedure in other gastrointestinal (GI) diseases, such as IBD without CDI, generally fails to effect cure. To improve results in treating other non-CDI diseases, innovatively designed Randomized Controlled Trials (RCTs) will be required to address questions about mechanisms operating within particular diseases. Availability of orally deliverable FMT products, such as capsules containing lyophilised fecal microbiota, will simplify CDI treatment and open the door to convenient, prolonged FMT delivery to the GI tract and will likely deliver improved results in both CDI and non-CDI diseases.
\end{abstract}

Keywords: Fecal microbiota transplantation; Clostridium difficile; inflammatory bowel disease; IBD; colitis; Crohn's; irritable bowel 


\section{Introduction}

This review avoids detailed summary about Fecal Microbiota Transplantation (FMT), a procedure that introduces normal donor colonic microbiota in the form of blended stool into a recipient's bowel to treat recurrent Clostridium difficile infection (CDI). The efficacy of FMT to treat recurrent CDI was initially proven in a randomized controlled trial by Van Nood et al. [1], where the study was halted due to the overwhelming success of donor FMT compared with vancomycin. Here, we reference "headline" topics in FMT in the Introduction, focusing primarily on CDI situations facing clinicians, questions arising among research physicians and emerging changes in compositions and delivery formats of transplantation material for FMT.

Since its first detailed description in 1958 by Ben Eiseman [2] using FMT in staphylococcal pseudomembranous enterocolitis, retrospectively thought to be CDI, FMT has matured through various stages. This process has involved the following:

- Early Case Reports: The "discovery phase" stemmed from a number of literature reports, often expressing considerable surprise that FMT worked so well to dramatically cure terminally ill patients. The severe condition was thought to be a staphylococcal pseudomembranous enterocolitis [2-5], but after 1981 [6,7], when CDI toxin became detectable [3-7], clinicians questioned and minimized the role of Staphylococcus aureus in pseudomembranous intestinal lesions because they realized that most pseudomembranous enterocolitis diagnoses were positive for CDI toxin [8].

- Donor Selection: Half a century later, with FMT becoming used more frequently, a group experienced in utilising FMT published the first standardized donor selection guidelines [9]. More stringent selection criteria are already appearing, and there is every reason to believe that more refined guidelines will emerge as we incorporate additional exclusion criteria, such as family history of obesity, metabolic syndrome and diabetes [10], into FMT practice.

- Methodology Refinements: As FMT case reports publish from various parts of the world, summaries and comparisons of numerous methodological analyses are helping refine FMT practice [11-14]. Topics reviewed to date include history of FMT, the changing face of CDI and stool solvent, volume and delivery routes (via the upper GI tract versus the lower GI tract). For example, and perhaps significant for future FMT administration, a recent study reported 27 patients who underwent fixed volume FMT delivered via upper enteroscopy and colonoscopy at the same session to cover both the small and large intestine. In the largest study of its kind, all 27 patients cleared $C$. difficile toxin from their systems, resulting in 100\% clinical efficacy with only minimal, transient adverse effects [15]. These cure rates contrast with that of $73 \%-92 \%$ for delivery via nasogastric/duodenal tubes and via upper endoscopy. This "dual coverage" method points to the importance of developing encapsulated microbiota to treat CDI, so that normal microbiota can be administered via oral delivery, thereby exposing the entire GI tract to healthy, diverse flora and potentially improving efficacy of CDI eradication.

- Long-Term Follow-up: Several articles have addressed the need for long-term follow up of patients receiving FMT since it is crucial to know about any untoward effects that may only develop long after the procedure [14,16]. At this time, short-term adverse effects have been remarkably infrequent following FMT [11]. In a long-term follow-up study of 77 patients after 
FMT, some new diseases developed in 4 subjects, although these had not been present in the donors and a clear relationship between the new disease and FMT was not evident [17]. Reflecting on the origin of the microbiota used in FMT, one could conclude that this "biologic" therapy derived from a healthy donor, say 35 years of age, has already undergone multiple decades of in vivo "testing" for adverse events within that healthy donor [18]. Long-term safety evidence to date [17] implies that microbiota from healthy, well-screened donors is currently the safest FMT product we have, but longitudinal studies to monitor and correlate gut microbiota with the health of donors and recipients have yet to be done. Indeed, in our own practice with 26 years follow-up of several thousand FMT recipients, there has been no donor-to recipient illness transfer. Ironically, the perceived safety of cultured consortia of defined microbiota, with their inherent potential for gene transfer and exchange, cannot rival the deep level of safety that comes from a healthy, well-screened human donor. Such data come from treating with non-toxigenic $C$. difficile to outcompete toxigenic strains to prevent colonization [19]. The C. difficile toxin-encoding PaLoc region from a toxigenic strain was recently shown to mobilize to non-toxigenic isolates, indicating that non-toxigenic strains can become toxigenic through horizontal gene transfer events [20]. Using spores of non-toxigenic strains, in this case Clostridia spores, in therapeutics may be risky, as evidenced by the fact that several placebo patients were found to be infected with non-toxigenic strains during clinical trials, apparently due to spore contamination of communal areas [21].

- Regrowth of Depleted Microbiota: Pre- and post-FMT microbiota composition of the recipient's GI tract has also been of particular interest. While it is possible to achieve durable implantation of donor bacteria [22], it is also becoming clear that eradicating $C$. difficile by antagonistic, but non-pathogenic, Clostridium spores can lead to a recovered, functional microbiota population due to regrowth of occult "missing" components such as Firmicutes and Bacteroidetes [23].

- FDA Oversight: In March 2014, the United States Food and Drug Administration (FDA) announced its intention to exercise enforcement discretion regarding Investigational New Drug (IND) applications for use of FMT for recurrent CDI. An IND application is not required to treat recurrent CDI cases, but it is still necessary for non-CDI indications and for research situations. Understanding the FDA position is crucial both to clinicians and FMT research teams [24].

\section{Expanding Use of FMT for CDI in Specialised Clinical Situations}

As more hospitals and clinics have begun using FMT to treat CDI, questions of safety and efficacy have arisen about special patient groups. We address these below using individual publications that combine cases from several practitioners.

- Elderly Patients: Elderly patients are the most susceptible to relapse after initial treatment of CDI with standard of care antibiotic therapy $[25,26]$ and are the majority of patients currently treated for relapsing CDI. Consequently, it is important to know that FMT is safe and effective in this patient population. Using data collected from multiple centres, Agrawal et al. [27] reported on 146 patients, finding $83.5 \%$ and $95.2 \%$ primary and secondary cure rates in relapsing CDI. These rates associated with a short-term adverse effect rate of $3.4 \%$ either due to the CDI, the FMT or both. 
- Patients with Severe CDI: The only published study with indices about this indication is a collection of 13 cases from a multicentre study of severe and/or complicated CDI in patients who had failed several courses of antibiotics and who were subsequently treated with FMT. Of these, $84 \%$ had severe CDI and $92 \%$ had complicated CDI, and their mean post-treatment follow-up was 15 months. Primary and secondary cure rates were $84 \%$ and $92 \%$, respectively, with minimal adverse effects of abdominal bloating and cramping early post treatment [28]. Such data indicate that age and severity should not be a barrier when considering FMT as a treatment option in the elderly, even those with severe and complicated CDI.

- The Immunosuppressed: This unique patient group frequently contracts CDI, and concerns have arisen regarding the safety of FMT for immunosuppressed patients with IBD and non-IBD associated-CDI given the possibility that septicaemia could result from the FMT procedure. In a retrospective multicentre study, Kelly et al. [29] reported on 80 CDI patients, each immunosuppressed due to HIV, solid organ transplant or cancer, and 36 of whom also had IBD. All patients received FMT, resulting in an $89 \%$ cure rate of CDI without any infection resulting from the FMT. They recorded several treatment-related adverse effects, including sedation-related aspiration and worsening of IBD. Brandt et al. [30] reported on a smaller cohort of 13 immunosuppressed IBD patients who had no CDI but were being treated for IBD with FMT. Apart from transient abdominal distension and bloating in 2 patients, there were no other adverse effects. Such data reinforce the conclusion that immunosuppressed patients have no increased risk of infection from the FMT treatment itself relative to non-immunosuppressed patients.

- Patients after Sub-total/total Colectomy: There are, as yet, few publications to indicate whether patients with partial or total colectomy suffering CDI are more difficult to cure with FMT than other types of CDI patients. At the Centre for Digestive Diseases (CDD), we have had a total of 3 patients with sub-total colectomy for whom FMT failed to cure their CDI, even with combined, repeated nasojejunal and colonic transcolonoscopic infusions; these patients have continued to have CDI for up to 7 years. There has been one publication [31], however, in which FMT via nasoduodenal administration cured a patient after total proctocolectomy of CDI in the remaining small bowel.

- Patients with IBD and CDI: A proportion of patients with ulcerative colitis (UC) and Crohn's disease (CD) are co-infected with Clostridium difficile. We have reported treating such patients with FMT, noting efficient eradication of the CDI, but there are several outcomes for the IBD $[32,33]$. IBD symptoms may improve initially in a sub-group for the first few weeks/month, followed by symptom recurrence. In a larger proportion of patients, symptoms remain unchanged, and, in a small percentage, the IBD symptoms worsen, perhaps due to the withdrawal of antibiotics in patients who receive FMT (to avoid interfere with the implanted bacteria). Others have reported similar observations [34]. 


\section{Expanding the Use of FMT to Non-CDI Colitis}

Clostridium difficile can be eradicated in patients with IBD even though the IBD is rarely cured. Whilst occasionally curable, IBD can respond to FMT, especially if the procedure is administered repeatedly, and can result in a "remission". In 1988, we administered FMT to a patient at CDD for colitis in the absence of CDI - the first of such patients to receive FMT at our facility. Her indeterminate colitis completely disappeared over several weeks and has not recurred over the past 26 years of follow-up [35,36]. We term such profound IBD remission as a "Sporadic Remission" after FMT. Figure 1 documents a more recent example of a CDD patient who had 14 days of FMT, after which her colitis reversed completely to normality for 3 years even though she did not have CDI. Based upon our extended experience over 24 years of using FMT in colitis patients [37], we believe that FMT researchers, as a group, can modify treatment paradigms to achieve better cure results and not just short term remissions. The first randomized clinical trials of FMT in Ulcerative Colitis (UC) have now been published [38,39]. Moayyedi et al. [38] reported significant induction of remission with FMT in UC where 75 patients received either a $50 \mathrm{~mL}$ FMT via enema $(\mathrm{n}=38)$ or $50 \mathrm{~mL}$ water enema $(\mathrm{n}=37)$ once weekly for six weeks. By week 7, 9/38 patients on FMT (24\%) were in remission due to "donor effect" while only 2/37 on placebo $(5 \%)$ were in remission $(p<0.03)$. In the second trial by Rossen et al. [39], patients received FMT via nasoduodenal tube at 0 and 3 weeks from either a donor (active arm) or their own stool (control arm). On intention-to-treat analysis, 7/23 on active (30.4\%) and 5 of 25 controls (20.0\%) achieved the primary endpoint $(p=0.51)$. Moayyedi achieved remission in spite of low FMT volumes, but his group did use frequent infusions via enema route. Rossen may have failed because only two infusions were carried out, and even these were via nasoduodenal route unlike the previously published UC methods, reflecting a trial design influenced by their previous CDI results [39]. Both trials measured attainment of remission rather than cure, yet they were testing a CDI therapy that measures cure rather than remission.

There is a fundamental difference between treating relapsing CDI with FMT and treating IBD with FMT. Relapsing CDI clearly has a different pathogenic mechanism than IBD, and outcome after FMT differs significantly between these two conditions. Perhaps in part due to publications documenting dramatic examples of sporadic remission in IBD after FMT (often actual cure of IBD [40]), pressure has been building to develop protocols, find funding and conduct randomized controlled trials (RCTs) for FMT in IBD. As recently noted, "the sparse results reported for cases of IBD have been variable with regard to the success rate for inducing remission, and well-designed randomized controlled trials are currently still lacking" [41]. RCTs for CDI aim to obtain cure with a single or several FMT infusions compared to placebo. Regrettably, not even well-designed RCTs will provide answers concerning IBD 'cure' until we throw out the CDI rule book and pose the correct questions for IBD. RCTs for IBD can be designed to give short-term remission following repeated administrations compared to currently used drugs such as steroids, but this approach does not result in prolonged remission. Alternatively, and taking "the high road", we can try to emulate CDI results and maximize IBD cure as in Figure 1. As of late April 2015, there were at least 23 active trials listed on www.clinicaltrials.gov comparing FMT with placebo in IBD (Table 1). We predict that many of these trials will fail to show a significant difference between FMT and placebo, as seen in Rossen 2015 [39], because the intervention protocol used is that for CDI. 
(a)
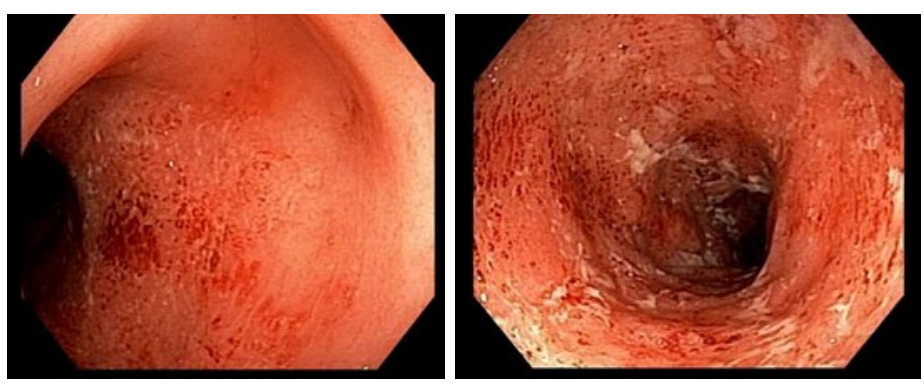

(b)
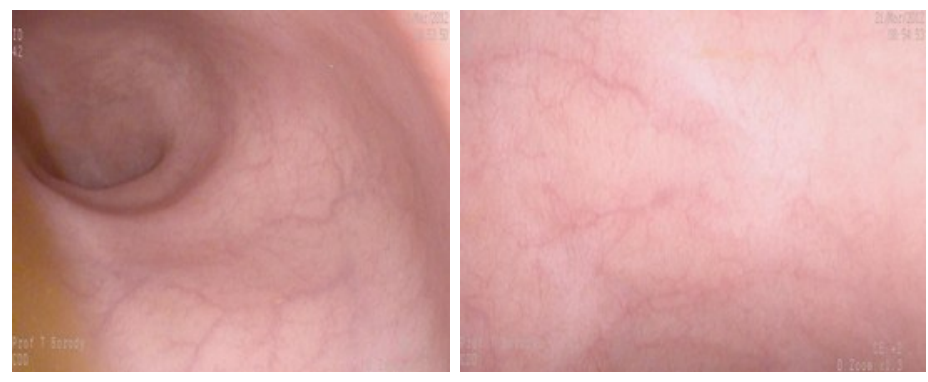

Figure 1. Sporadic "remission" of ulcerative colitis after 14 days of Fecal Microbiota Transplantation (FMT) showing mucosa before and three years after treatment. Patient off medications. (a) BEFORE FMT: Marked inflammation in rectum and sigmoid colon; (b) AFTER FMT: Absence of inflammation in rectum and sigmoid colon.

Table 1. Current and upcoming clinical trials targeting IBD and related illnesses with FMT

\begin{tabular}{ccc}
\hline Clinicaltrials.gov Identifier & Indication being Trialed & Phase of Trial \\
\hline NCT01790061 & UC & Phase 2/Phase 3 \\
NCT01793831 & CrD & Phase 2/Phase 3 \\
NCT01847170 & IBD & Phase 1 \\
NCT01896635 & UC & Phase 2 \\
NCT01947101 & UC & Phase 1 \\
NCT02016469 & IBD & NP \\
NCT02033408 & IBD & Phase 4 \\
NCT02049502 & UC-associated Pouchitis & Phase 2 \\
NCT02058524 & UC & Phase 1 \\
NCT02092402 & IBS & NP \\
NCT02108821 & IBD & Phase 1 \\
NCT02154867 & IBS & Phase 2 \\
NCT02199561 & CrD & Phase1/Phase 2 \\
NCT02227342 & UC & Phase 1/Phase 2 \\
NCT02291523 & UC & NP \\
NCT02299973 & IBS & NP \\
NCT02328547 & IBS & Phase 2 \\
NCT02330211 & Crohn's Colitis & Phase1/Phase 2 \\
NCT02330653 & UC & Phase1/Phase 2 \\
NCT02335281 & IBD & Phase 2 \\
NCT02391012 & IBD & Phase 1 \\
NCT02417974 & CrD & Phase 2 \\
NCT02390726 & UC & Phase 0 \\
\hline
\end{tabular}

Abbreviations List: CDI-Clostridium difficile Infection, CrD-Crohn's Disease, IBD-Irritable Bowel Disease, IBS-Irritable Bowel Syndrome, NP-Not Provided on clinicaltrials.gov, UC-Ulcerative Colitis 
Hence, the alternative is to design FMT trials for IBD that address questions about what methods will improve FMT therapy to achieve more "sporadic remissions" of IBD. A clear example of such evolving methodology occurred when Dr. Patrizia Kump and colleagues initially trialed single FMT for UC and found no clinical improvement [42]. Later they used multiple FMT infusions, which achieved significant improvement from baseline [43], and noted that serially repeated FMT produced better efficacy than a single FMT [43]. Such informed trial designs are needed to drive improved outcomes. Issues to address may include the following:

a. Number and frequency of FMTs

b. Use of frozen stool vs. fresh stool vs. a selected consortium of organisms

c. Pre-treatment with antibiotics, including which antibiotics, how many antibiotics and how long to pre-treat with antibiotics

d. Determining whether there are more/less efficacious donor microbiota and what compositional differences affect efficacy

e. Determining whether to administer FMT during active mucosal inflammation or to heal the mucosa with anti-inflammatory medications, e.g., immunosuppressive therapy, and then administer FMT

f. Determining whether to maintain mucosal healing therapy after FMT, e.g., with 6-mercaptopurine or azathioprine for prolonged periods of time, while waiting for the transplanted microbiota to effect the "healing process" and, perhaps, to improve implantation? In a study by Borody et al. [37], patient follow up at 33 months revealed that $57 \%$ of the patients achieved mucosal healing when some were maintained on 6-mercaptopurine or azathioprine after FMT. This suggests that long-term follow-up of these patients, some of whom could be kept on anti-inflammatory agents, could be yet another mechanism by which to increase the success rate of FMT for UC patients.

\section{FMT Use in Non-CDI Single Infections}

Based on the success of FMT in treating CDI, clinicians have begun using FMT to treat single bacterial infections distinct from CDI. Singh et al. [44] recently described using FMT to eradicate an Extended Spectrum beta-Lactamase (ESBL) producing Escherichia coli from the colon of a renal transplant patient, leading to cessation of the patient's recurrent urinary infection with this pathogen. We agree with the authors' hypothesis that donor feces infusion can be effective against pathogens other than C. difficile, such as the ESBL-producing Enterobacteriacae of the large bowel. Due to the success they observed in this case, the authors have initiated a clinical trial using FMT in patients infected with ESBL-producing Enterobacteriacae.

A second infectious agent treated successfully by FMT was reported by Freedman in 2014 [45]. In this report presented at the 2014 Infectious Diseases Week meeting in Philadelphia, Freedman described multi-drug resistant carbapenemase-producing Klesbiella pneumoniae residing in the bowel that caused repeated blood, joint and femur osteomyelitis infections in a 13-year-old girl. After all antibiotic treatments failed, FMT cleared her gut of this Klebsiella and further systemic infections over the next 5 years. This experience indicates that FMT can cure GI pathogens other than $C$. difficile and that such pathogenic bacteria may be a source of systemic infection. 


\section{Microbiome-Derived FMT Therapies Moving to Mainstream Medicine}

We believe that a broad array of microbiome-derived, or microbiome-inspired, therapeutics will be developed over the next decade for numerous indications, including both gastrointestinal and systemic disorders and diseases. The ability to cure recurrent Clostridium difficile infection (R-CDI) safely and effectively with FMT in a clinical setting has spurred several companies to pursue FDA-approvable therapeutics to treat that indication. To date these therapeutic candidates, which all, to the best of our knowledge, derive from the stool of selected, extensively tested healthy human donors, fall into three categories: (1) homogenized and minimally refined whole stool, thereby comprising a "full-spectrum" of gastrointestinal microbiota, (2) highly concentrated and quantitated "full-spectrum" microbiota and (3) highly concentrated and quantitated "narrow-spectrum" spores and/or vegetative cells. Table 2 lists current product candidates, the targeted routes of administration, and companies developing the products. The Office of Vaccines Research and Review (OVRR) within the FDA's Center for Biologics Evaluation and Research (CBER) will evaluate each candidate as a biologic.

Table 2. Current FMT product candidates for various conditions.

\begin{tabular}{ccc}
\hline Product Candidate & Route of Administration & Company \\
\hline MB-101 & Oral delivery & Assembly Biosciences ${ }^{1}$ \\
Full-spectrum Microbiota ${ }^{\mathrm{TM}}$ & Oral, colonoscopic delivery & CIPAC Limited \\
RBX2660 & Enema delivery & Rebiotix Inc \\
Ecobiotic ${ }^{\circledR}$ SER-109 & Oral delivery & Seres Health \\
\hline
\end{tabular}

${ }^{1}$ Collaborating with OpenBiome, a nonprofit 501(c)(3) organization

In 2012, Hamilton et al. [46] reported on 43 consecutive patients with recurrent CDI who were treated with fresh or frozen filtered material prepared as fecal bacteria extract. Following a single infusion, $90 \%$ of patients administered frozen donor material were cured of CDI. A preliminary study performed by Youngster et al. [47] assessed the safety and efficacy of encapsulated frozen FMT for relapsing CDI. Resolution of diarrhea was observed in $70 \%$ of patients after a single capsule-based FMT, with non-responders retreated, to achieve an overall $90 \%$ rate of clinical resolution of diarrhea. No serious adverse events were reported, and only minor transient events were noted, including mild abdominal bloating and cramping in $30 \%$ of patients, which resolved within 72 hours. While the bulk of material used for FMT procedures to date has been crude or refined suspensions of fresh or frozen fecal microbiota, issues of convenience and stability clearly demand development of lyophilised preparations as approved therapeutic products.

Lyophilised product candidates to treat R-CDI are being developed in both the full-spectrum and narrow-spectrum categories; such formulations could be resuspended for nasogastric or rectal (enema or colonoscopy) delivery or encapsulated for oral administration. Encapsulated, orally administered microbiota therapies - whether narrow- or full-spectrum - will facilitate treating recurrent $C$. difficile infection and will expand our ability to explore safety and efficacy in other, non-CDI indications. Given the documented efficacy of fecal microbiota in curing R-CDI and non-CDI single infections, the positive - albeit inconsistent - effects on UC and Crohn's disease and the growing awareness about the systemic importance of healthy gut microbiota [48], future clinical trials will likely explore microbiome-based therapies for a variety of other, non-gastrointestinal conditions. 
Susceptibility to obesity, liver disease, cardiovascular disease and malignancy correlate with gut microbiota dysbiosis [17], and specific bacterial populations have been identified in a variety of other disorders. For example, Longstreth et al. [19] have hypothesized that a motor neuron toxin produced by a Clostridial species causes sporadic amyotrophic lateral sclerosis (ALS) in susceptible individuals, Scher et al. [20] have found that an expanded population of intestinal Prevotella copri correlates with enhanced susceptibility to arthritis, while Hsiao et al. [21] have determined that gut microbiota modulate behavioral and physiological abnormalities associated with neurodevelopmental disorders such as autism spectrum disorder (ASD).

\section{Conclusions}

The use of FMT in CDI has become rapidly accepted as a simple yet effective therapy that is becoming frequently utilized by the medical community. Success in treating CDI with healthy microbiota has created a natural extension into exploring FMT therapy for other gastrointestinal conditions, and the relevance of a robust gut microbiome to general health has generated significant interest in evaluating FMT therapy for a variety of systemic, non-gastrointestinal disorders. We now need to stop, reflect, and develop ways to harness the power of a therapy that might be capable of achieving in non-CDI conditions the success it enjoys in CDI. Orally available microbiota-based products, several of which are being developed currently, will facilitate treatment for Clostridium difficile infection and further expand the horizons of exciting therapeutic opportunities for numerous other non-CDI indications.

\section{Author Contributions}

Thomas Borody conceived of this review, and he, Debra Peattie and Scott Mitchell collaborated in writing and editing the manuscript.

\section{Conflicts of Interest}

Thomas Borody has a financial interest in the Centre for Digestive Diseases (New South Wales, Australia), where fecal microbiota transplant is presented as a treatment option. Additionally, he has filed patent applications in this field and is a consultant for CIPAC Limited, a start-up company developing microbiome-based therapeutics.

Debra Peattie is Managing Director of Pleiades Advisors, a privately held firm that offers strategic advising services to life science companies, including CIPAC Limited, a start-up company developing microbiome-based therapeutics.

Scott Mitchell is an employee of the Centre for Digestive Diseases (New South Wales, Australia) where fecal microbiota transplant is offered as a treatment.

Apart from those disclosed above, the authors have no relevant affiliations with any organization or entity with a financial interest in or conflict with the subject matter or materials discussed in the manuscript.

The authors utilized no writing assistance in producing this manuscript. 


\section{References}

1. Van Nood, E.; Vrieze, A.; Nieuwdorp, M.; Fuentes, S.; Zoetendal, E.G.; de Vos, W.M.; Visser, C.E.; Kuijper, E.J.; Bartelsman, J.F.; Tijssen, J.G.; et al. Duodenal infusion of donor feces for recurrent Clostridium difficile. N. Engl. J. Med. 2013, 368, 407-415.

2. Eiseman, B.; Silen, W.; Bascom, G.S.; Kauvar, A.J. Fecal enema as an adjunct in the treatment of pseudomembranous enterocolitis. Surgery 1958, 44, 854-859.

3. Collins, D.C. Pseudomembranous enterocolitis. Further observations on the value of donor fecal enemata as an adjunct in the treatment of pseudomembranous enterocolitis. Am. J. Proctol. 1960, 2, 389-391.

4. Cutolo, L.C.; Kleppel, N.H.; Freund, H.R.; Holker, J. Fecal feedings as a therapy in Staphylococcus enterocolitis. NY State J. Med. 1959, 59, 3831-3833.

5. Fenton, S.; Stephenson, D.; Weder, C. Pseudomembranous colitis associated with antibiotic therapy-An emerging entity. Can. Med. Assoc. J. 1974, 111, 1110-1111, 1114.

6. Bowden, T.A., Jr.; Mansberger, A.R., Jr.; Lykins, L.E. Pseudomembraneous enterocolitis: Mechanism for restoring floral homeostasis. Am. Surg. 1981, 47, 178-183.

7. Schwan, A.; Sjolin, S.; Trottestam, U.; Aronsson, B. Relapsing Clostridium difficile enterocolitis cured by rectal infusion of homologous faeces. Lancet 1983, 2, 845.

8. Froberg, M.K.; Palavecino, E.; Dykoski, R.; Gerding, D.N.; Peterson, L.R.; Johnson, S. Staphylococcus aureus and Clostridium difficile cause distinct pseudomembranous intestinal diseases. Clin. Infect. Dis. 2004, 39, 747-750.

9. Bakken, J.S.; Borody, T.; Brandt, L.J.; Brill, J.V.; Demarco, D.C.; Franzos, M.A.; Kelly, C.; Khoruts, A.; Louie, T.; Martinelli, L.P.; et al. Treating Clostridium difficile infection with fecal microbiota transplantation. Clin. Gastroenterol Hepatol. 2011, 9, 1044-1049.

10. Alang, N.; Kelly, C.R. Weight gain after fecal microbiota transplantation. Open Forum Infect. Dis. 2015, doi:10.1093/ofid/ofv004.

11. Gough, E.; Shaikh, H.; Manges, A.R. Systematic review of intestinal microbiota transplantation (fecal bacteriotherapy) for recurrent Clostridium difficile infection. Clin. Infect. Dis. 2011, 53, 994-1002.

12. Borody, T.; Leis, S.; Pang, G.; Wettstein, A. Fecal microbiota transplantation in the treatment of recurrent Clostridium difficile infection. Available online: http://www.uptodate.com/contents/fecalmicrobiota-transplantation-in-the-treatment-of-recurrent-clostridium-difficile-infection (accessed on 26 April 2015).

13. Landy, J.; Al-Hassi, H.O.; McLaughlin, S.D.; Walker, A.W.; Ciclitira, P.J.; Nicholls, R.J.; Clark, S.K.; Hart, A.L. Review article: Faecal transplantation therapy for gastrointestinal disease. Aliment. Pharmacol. Ther. 2011, 34, 409-415.

14. Brandt, L.J. American journal of gastroenterology lecture: Intestinal microbiota and the role of fecal microbiota transplant (FMT) in treatment of C. difficile infection. Am. J. Gastroenterol. 2013, 108, $177-185$. 
15. Dutta, S.K.; Girotra, M.; Garg, S.; Dutta, A.; von Rosenvinge, E.C.; Maddox, C.; Song, Y.; Bartlett, J.G.; Vinayek, R.; Fricke, W.F. Efficacy of combined jejunal and colonic fecal microbiota transplantation for recurrent Clostridium difficile infection. Clin. Gastroenterol. Hepatol. 2014, 12, 1572-1576.

16. Brandt, L.J.; Aroniadis, O.C.; Mellow, M.; Kanatzar, A.; Kelly, C.; Park, T.; Stollman, N.; Rohlke, F.; Surawicz, C. Long-term follow-up of colonoscopic fecal microbiota transplant for recurrent Clostridium difficile infection. Am. J. Gastroenterol. 2012, 107, 1079-1087.

17. Blumberg, R.; Powrie, F. Microbiota, disease, and back to health: A metastable journey. Sci. Transl. Med. 2012, 4, doi:10.1126/scitranslmed.3004184.

18. Borody, T.J.; Peattie, D.; Kapur, A. Could fecal microbiota transplantation cure all Clostridium difficile infections? Future Microbiol. 2014, 9, 1-3.

19. Longstreth, W.T., Jr.; Meschke, J.S.; Davidson, S.K.; Smoot, L.M.; Smoot, J.C.; Koepsell, T.D. Hypothesis: A motor neuron toxin produced by a clostridial species residing in gut causes ALS. Med. Hypotheses 2005, 64, 1153-1156.

20. Scher, J.U.; Sczesnak, A.; Longman, R.S.; Segata, N.; Ubeda, C.; Bielski, C.; Rostron, T.; Cerundolo, V.; Pamer, E.G.; Abramson, S.B.; et al. Expansion of intestinal Prevotella copri correlates with enhanced susceptibility to arthritis. Elife 2013, 2, e01202.

21. Hsiao, E.Y.; McBride, S.W.; Hsien, S.; Sharon, G.; Hyde, E.R.; McCue, T.; Codelli, J.A.; Chow, J.; Reisman, S.E.; Petrosino, J.F.; et al. Microbiota modulate behavioral and physiological abnormalities associated with neurodevelopmental disorders. Cell 2013, 155, 1451-1463.

22. Grehan, M.J.; Borody, T.J.; Leis, S.M.; Campbell, J.; Mitchell, H.; Wettstein, A. Durable alteration of the colonic microbiota by the administration of donor fecal flora. J. Clin. Gastroenterol. 2010, 44, 551-561.

23. Khanna, S.; Pardi, D.S.; Kelly, C.; Pindar, C.; Kammer, D.; McKenney, J.; Vuliv, M.; Lombardo, M.; Henn, M.; Aunins, J.; et al. Clinical evaluation of ser-109, a rationally designed, oral microbiomebased therapeutic for the treatment of recurrent Clostridium difficile. Available online: http://www.gastro.org/pressroom/James_W._Freston_Abstract_Orally_Delivered_Microbes.pdf (accessed on 26 April 2015).

24. Kelly, C.R.; Kunde, S.S.; Khoruts, A. Guidance on preparing an investigational new drug application for fecal microbiota transplantation studies. Clin. Gastroenterol. Hepatol. 2014, 12, 283-288.

25. Pepin, J.; Alary, M.E.; Valiquette, L.; Raiche, E.; Ruel, J.; Fulop, K.; Godin, D.; Bourassa, C. Increasing risk of relapse after treatment of Clostridium difficile colitis in Quebec, Canada. Clin. Infect. Dis. 2005, 40, 1591-1597.

26. Lessa, F.C.; Mu, Y.; Bamberg, W.M.; Beldavs, Z.G.; Dumyati, G.K.; Dunn, J.R.; Farley, M.M.; Holzbauer, S.M.; Meek, J.I.; Phipps, E.C.; et al. Burden of Clostridium difficile infection in the United States. N. Engl. J. Med. 2015, 372, 825-834.

27. Agrawal, M.; Aroniadis, O.C.; Brandt, L.J.; Kelly, C.; Freeman, S.; Surawicz, C.; Broussard, E.; Stollman, N.; Giovanelli, A.; Smith, B.A. A long-term follow-up study of the efficacy and safety of fecal microbiota transplant (FMT) for recurrent/severe/complicated $C$. difficile infection (CDI) in the elderly. Gastroenterology 2014, 146, doi:10.1016/S0016-5085(14)60147-5. 
28. Aroniadis, O.C.; Brandt, L.J.; Greenberg, A.; Borody, T.J.; Kelly, C.; Mellow, M.; Surawicz, C.; Cagle, L.A.; Neshatian, L. Long-term follow-up study of fecal microbiota transplantation (FMT) for severe or complicated Clostridium difficile infection (CDI). Gastroenterology 2013, 144, S185.

29. Kelly, C.R.; Ihunnah, C.; Fischer, M.; Khoruts, A.; Surawicz, C.; Afzali, A.; Aroniadis, O.; Barto, A.; Borody, T.; Giovanelli, A.; et al. Fecal microbiota transplant for treatment of Clostridium difficile infection in immunocompromised patients. Am. J. Gastroenterol. 2014, 109, 1065-1071.

30. Brandt, L.; Aroniadis, O.; Greenberg, A.; Borody, T.; Finlayson, S.; Furnari, V.; Kelly, C. Safety of fecal microbiota transplantation (FMT) in immunocompromised (IC) patients with inflammatory bowel disease (IBD). Am. J. Gastroenterol. 2013, 108, S556-S556.

31. Miao, C.L.; Mowery, A.D.; Khara, H.S.; Shellenberger, M.J.; Komar, M. C. Difficile small bowel enteritis after total proctocolectomy successfully treated with fecal transplant. Presented at the American College of Gastroenterologists, Philadelphia, PA, USA, 20 October 2014.

32. Borody, T.J.; Wettstein, A.R.; Leis, S.; Hills, L.A.; Campbell, J.; Torres, M. Clostridium difficile complicating inflammatory bowel disease: Pre-and post-treatment findings. Gastroenterology 2008, 134, A361.

33. Borody, T.; Wettstein, A.; Nowak, A. Fecal microbiota transplantation (FMT) eradicates Clostridium difficile infection (CDI) in inflammatory bowel disease (IBD). UEG J. 2013, 1, A57.

34. Singh, N.; Suskind, D.; Wahbeh, G. Fecal bacteriotherapy in a 6 year old patient with ulcerative colitis and Clostridium difficile: P-136 yi. Inflamm. Bowel. Dis. 2012, 18, S69.

35. Borody, T.J.; George, L.; Andrews, P.; Brandl, S.; Noonan, S.; Cole, P.; Hyland, L.; Morgan, A.; Maysey, J.; Moore-Jones, D. Bowel-flora alteration: A potential cure for inflammatory bowel disease and irritable bowel syndrome? Med. J. Aust. 1989, 150, 604.

36. Borody, T.J.; Campbell, J. Fecal microbiota transplantation: Techniques, applications, and issues. Gastroenterol. Clin. North Am. 2012, 41, 781-803.

37. Borody, T.; Wettstein, A.; Campbell, J.; Leis, S.; Torres, M.; Finlayson, S.; Nowak, A. Fecal microbiota transplantation in ulcerative colitis: Review of 24 years experience. Am. J. Gastroenterol. 2012, 107, S665-S665.

38. Moayyedi, P.; Surette, M.G.; Kim, P.T.; Libertucci, J.; Wolfe, M.; Onischi, C.; Armstrong, D.; Marshall, J.K.; Kassam, Z.; Reinisch, W.; et al. Fecal microbiota transplantation induces remission in patients with active ulcerative colitis in a randomized controlled trial. Gastroenterology 2015, $149,102-106$.

39. Rossen, N.G.; Fuentes, S.; van der Spek, M.J.; Tijssen, J.; Hartman, J.H.; Duflou, A.; Lowenberg, M.; van den Brink, G.R.; Mathus-Vliegen, E.M.; de Vos, W.M.; et al. Findings from a randomized controlled trial of fecal transplantation for patients with ulcerative colitis. Gastroenterology 2015, $149,110-118$.

40. Zhang, F.M.; Wang, H.G.; Wang, M.; Cui, B.T.; Fan, Z.N.; Ji, G.Z. Fecal microbiota transplantation for severe enterocolonic fistulizing crohn's disease. World J. Gastroenterol. 2013, 19, 7213-7216.

41. Kostic, A.D.; Xavier, R.J.; Gevers, D. The microbiome in inflammatory bowel disease: Current status and the future ahead. Gastroenterology 2014, 146, 1489-1499.

42. Kump, P.; Gröchenig, H.; Spindelböck, W.; Hoffmann, C.; Gorkiewicz, G.; Wenzl, H. Preliminary clinical results of repeatedly fecal microbiota transplantation (FMT) in chronic active ulcerative colitis. United European Gastroenterol. J. 2013, 1, A57. 
43. Kump, P.K.; Grochenig, H.P.; Lackner, S.; Trajanoski, S.; Reicht, G.; Hoffmann, K.M.; Deutschmann, A.; Wenzl, H.H.; Petritsch, W.; Krejs, G.J.; et al. Alteration of intestinal dysbiosis by fecal microbiota transplantation does not induce remission in patients with chronic active ulcerative colitis. Inflamm. Bowel. Dis. 2013, 19, 2155-2165.

44. Singh, R.; van Nood, E.; Nieuwdorp, M.; van Dam, B.; ten Berge, I.J.; Geerlings, S.E.; Bemelman, F.J. Donor feces infusion for eradication of extended spectrum beta-lactamase producing Escherichia coli in a patient with end stage renal disease. Clin. Microbiol. Infect. 2014, 20, 0977-O978.

45. Freedman, A. Use of Stool Transplant to Clear Fecal Colonization with Carbapenem-Resistant Enterobacteraciae (cre): Proof of Concept; Infectious Diseases Society of America: Philadelphia, PA, USA, 2014.

46. Hamilton, M.J.; Weingarden, A.R.; Sadowsky, M.J.; Khoruts, A. Standardized frozen preparation for transplantation of fecal microbiota for recurrent Clostridium difficile infection. Am. J. Gastroenterol. 2012, 107, 761-767.

47. Youngster, I.; Russell, G.H.; Pindar, C.; Ziv-Baran, T.; Sauk, J.; Hohmann, E.L. Oral, capsulized, frozen fecal microbiota transplantation for relapsing Clostridium difficile infection. JAMA 2014, 312, 1772-1778.

48. Smits, L.P.; Bouter, K.E.; de Vos, W.M.; Borody, T.J.; Nieuwdorp, M. Therapeutic potential of fecal microbiota transplantation. Gastroenterology 2013, 145, 946-953.

(C) 2015 by the authors; licensee MDPI, Basel, Switzerland. This article is an open access article distributed under the terms and conditions of the Creative Commons Attribution license (http://creativecommons.org/licenses/by/4.0/). 